\title{
Thermal radiation antennas made of multilayer structures containing negative index metamaterials
}

\author{
M. Maksimovic ${ }^{a}$, M. Hammer ${ }^{a}$, Z. Jaksic ${ }^{b}$ \\ ${ }^{a} \mathrm{MESA}^{+}$Institute for Nanotechnology, \\ University of Twente, The Netherlands \\ ${ }^{b}$ IHTM- Institute for Microelectronic Technologies and Single Crystals, \\ University of Belgrade, Serbia
}

\begin{abstract}
We investigate the thermal antenna behavior of emissive/absorptive substrates coated by passive optical multilayer systems that contain negative refractive index metamaterials (NIM). Spectral and angular distributions of the thermal radiation emittance for periodic defect-containing multilayer with NIM is addressed. We analyze realistic finite structures and took into account dispersion and losses in the NIM part. The application of NIM-containing 1D structures offers new degrees of freedom for the design, thus opening a path to obtain spectrally and spatially selective thermal emitters that could lead to improvements in the existing systems for thermal radiation control.
\end{abstract}

\section{INTRODUCTION}

One of the topics of interest in the electromagnetic optics of nanostructured media is the possibility to tailor emittance/absorptance by changing the distribution of electromagnetic modes. The theoretical foundation for the modification of thermal radiation by photonic bandgap materials (PBG) has been outlined in. ${ }^{1}$ The possibilities offered by periodic all-dielectric or metal-dielectric PBG media were expanded by the introduction of electromagnetic metamaterials with negative refractive index (NIM). ${ }^{2-6}$ Multilayers consisting of alternating dielectric (positive index material, PIM) and NIM layers able to offer unique design opportunities in emittance/absorptance tailoring. ${ }^{7-9}$ Widening and flattening of the spectral emittance was demonstrated, while at the same time the angular dependence of the NIM-containing multilayers proved to be much weaker. ${ }^{7}$ An especially interesting path to tailoring of emittance/absorptance was opened by utilizing NIM-containing periodic, ${ }^{7}$ fractal $^{8}$ and other quasiperiodic ${ }^{9}$ multilayers. The use of pre-fractal Cantor NIM-containing multilayers showed a super-directivity and spectral selectivity of the emittance. ${ }^{8}$ These properties are a consequence of the phase compensation effects ${ }^{10}$ associated with the presence of the NIM layers.

Ability of NIM-based structures for extended photonic bandgap engineering give rise to so-called zero-n bandgap appears when alternating PIM/NIM layers are stacked. ${ }^{11}$ It is a new type of photonic band gap, different from the Bragg gap, that occurs when the averaged effective refractive index of the whole structure equals zero. The zero-n gap is invariant to the length-scale, and almost insensitive to randomness, i.e. it persists for periodic, ${ }^{11}$ quasi-periodic ${ }^{12}$ and aperiodic structures. ${ }^{13}$

The design of thermal sources with their emittance enhanced in a narrow solid angle by applying multilayer filter structures has been of interest in the last few years. ${ }^{6,14-17}$ All structures so far used all-dielectric or metal-dielectric multilayer coatings to enhance or suppress thermal emission of absorptive/emissive substrates. ${ }^{15}$ These designs proved that it was practically feasible to obtain antenna-like behavior for thermal sources in the IR range. Thin film technology has been a sound foundation for this task. However, advances in the technology of nanostructured materials enabled the fabrication of materials with optical properties not readily found in nature, e.g. of NIMs for the optical range, see ${ }^{6}$ and references therein. This offers new possibilities for the device design.

In this paper we exploit the additional design freedom offered by the introduction of NIMs to implement a thermal emissivity control and an antenna-like response. We apply passive NIM-containing multilayers to tailor the spectral and angular emittance/absorptance distributions. On the basis of the transfer matrix approach and of the Kirchhoff's second law, we analyze realistic finite structures that comprise periodic defect-containing multilayers. Dispersion and losses in the NIM part are taken into account. 


\section{THEORY}

For the propagation of electromagnetic waves though planar layered structures made of piecewise constant, homogeneous, and isotropic media without sources, the vectorial wave equation reduces to two uncoupled scalar equations. ${ }^{18}$ One distinguishes two types of optical fields: For Transverse Electric (TE) waves the electric field is perpendicular to the plane spanned by the direction of propagation of the incident wave and its projection on the layer interfaces, while for Transverse Magnetic (TM) waves the magnetic field is perpendicular to that plane of incidence. Cartesian coordinates are introduced as in Figure 1 to describe the propagation of plane waves through the multilayer stacks, where the $x$-axis is parallel to the layer interfaces, while the $z$-axis is perpendicular to the stack surface, such that the coordinates $x$ and $z$ span the plane of incidence.

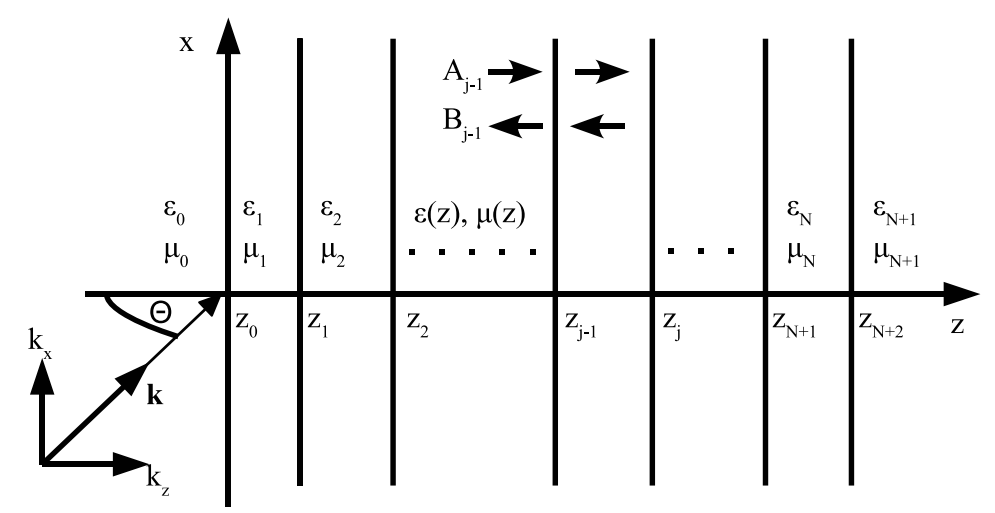

Figure 1. An inhomogeneous multilayer structure (i.e. a stratified medium) with piecewise constant, $z$-dependent permittivity $\epsilon$ and permeability $\mu$. The structure is invariant along the $x$ - and $y$-directions. Oblique incidence of plane electromagnetic waves is considered, with incidence angle $\theta$.

For TE waves the electric field $\boldsymbol{E}=\left(0, E_{y}, 0\right)$ is linearly polarized in the $y$-direction. Time-harmonic fields $\boldsymbol{E}(\boldsymbol{r}, t)=$ $\boldsymbol{E}(\boldsymbol{r}) \mathrm{e}^{-i \omega t}$ with real angular frequency $\omega$ are considered. Then under external time harmonic, TE polarized excitation the field in the medium is described by the scalar function $E_{y}(x, z)$ (where we drop the subscript ${ }_{y}$ to simplify the notation). With this choice of polarization the Maxwell equations reduce, after stratification $\partial_{y}=0$, to the Helmholtz equation for TE waves

$$
\left(\frac{\partial^{2}}{\partial x^{2}}+\mu(z) \frac{\partial}{\partial z} \frac{1}{\mu(z)} \frac{\partial}{\partial z}+\frac{\omega^{2}}{c^{2}} \epsilon \mu\right) E(x, z)=0 .
$$

Analogously, the principal magnetic component $H$ of time harmonic TM waves with $y$-polarized magnetic field $\boldsymbol{H}(x, z, t)=$ $(0, H, 0)(x, z) \mathrm{e}^{-i \omega t}$ satisfies the Helmholtz equation

$$
\left(\frac{\partial^{2}}{\partial x^{2}}+\epsilon(z) \frac{\partial}{\partial z} \frac{1}{\epsilon(z)} \frac{\partial}{\partial z}+\frac{\omega^{2}}{c^{2}} \epsilon \mu\right) H(x, z)=0
$$

Fourier transform along the layer interfaces separates the $x$ - and $z$-dependent parts of the principal fields, such that these can be represented in the form

$$
E(x, z)=E(z) \mathrm{e}^{ \pm i k_{x} x}, \text { and } H(x, z)=H(z) \mathrm{e}^{ \pm i k_{x} x}
$$

where the $x$-component $k_{x}$ of the wavevector now plays the role of a parameter that is defined by the angle of incidence (cf. Figure 1). Due to the invariance in the $x$-direction equations (1) and (2) become ordinary differential equations

$$
\left(\mu(z) \frac{\partial}{\partial z} \frac{1}{\mu(z)} \frac{\partial}{\partial z}+\frac{\omega^{2}}{c^{2}} \epsilon \mu-k_{x}^{2}\right) E(z)=0,
$$

and

$$
\left(\epsilon(z) \frac{\partial}{\partial z} \frac{1}{\epsilon(z)} \frac{\partial}{\partial z}+\frac{\omega^{2}}{c^{2}} \epsilon \mu-k_{x}^{2}\right) H(z)=0
$$


Note that these equations are identical for positions $z$ inside the layers with constant material properties. Differences between the polarizations manifest only through different interface conditions at the boundaries between the layers. For TE waves, the quantities

$$
E, \quad \frac{1}{\mu} \frac{\partial E}{\partial z}
$$

should be continuous across the interfaces, while continuity of

$$
H, \quad \frac{1}{\epsilon} \frac{\partial H}{\partial z}
$$

is required for TM polarized waves.

Analytic solutions of the Helmholtz equation for the multilayer structure of Figure 1 in the $j$-th layer can be written

$$
F_{j}(z)=A_{j} \mathrm{e}^{i k_{j z}\left(z-z_{j-1}\right)}+B_{j} \mathrm{e}^{-i k_{j z}\left(z-z_{j-1}\right)}
$$

where $F$ replaces the $E$-field in the case of TE polarization and the $H$-field for TM polarization. $k_{j z}$ is the $z$-component of the local wavevector in layer $j$, defined by

$$
k_{j z}^{2}=\frac{\omega^{2}}{c^{2}} \epsilon_{j} \mu_{j}-k_{x}^{2}
$$

for vacuum speed of light $c$.

We consider a situation when a plane wave $F_{0}(x, z)=A_{0} \mathrm{e}^{i k_{x} x+i k_{0 z} z}$ with given amplitude $A_{0}$ is incident onto the multilayer structure, coming from a semi-infinite, homogeneous medium, with wavevector $\boldsymbol{k}_{0 j}=\left(k_{x}, 0, k_{0 z}\right)$. Its $x$ - and $z$ components $k_{x}=\left(n_{0} \omega / c\right) \sin \theta$ and $k_{0 z}=\left(n_{0} \omega / c\right) \cos \theta$ define / are defined by the incidence angle $\theta$, where $n_{0}=\sqrt{\epsilon_{0} \mu_{0}}$ is the local refractive index. Local wavevector in $j$-th layer can be expressed as

$$
k_{j z}^{2}=\frac{\omega}{c} \sqrt{\epsilon_{j} \mu_{j}} \sqrt{1-\frac{n_{0}^{2} \sin ^{2} \theta}{n_{j}^{2}}}
$$

Inside the layer $z \in\left[z_{j-1}, z_{j}\right]$ with local permittivity $\epsilon_{j}$ and permeability $\mu_{j}$, the refractive index can be defined by $n_{j}=$ $\sqrt{\epsilon_{j} \mu_{j}}$. Particular care is required with the definition of the (sign of) the refractive index, in cases where the permittivity and permeability are complex quantities, with not necessarily positive real parts. The sign of the square root in the expression for refractive index determined according to the following rule:

$$
\begin{array}{ll}
\operatorname{Re}(n)<0 & \text { if } \operatorname{Re}(\epsilon)<0 \text { and } \operatorname{Re}(\mu)<0, \\
\operatorname{Re}(n) \geq 0 & \text { otherwise. }
\end{array}
$$

The term "negative index metamaterials" refers to situations where the first alternative of the rule (11) applies. The standard model for the absorption in the material is obtained by taking the complex form of the permittivity and permeability in corresponding layers. ${ }^{18,21}$ Then, for the complex material response functions, permeability $\epsilon(\omega)=\epsilon_{\text {re }}(\omega)+i \epsilon_{i m}(\omega)$ and permittivity $\mu(\omega)=\mu_{r e}(\omega)+i \mu_{i m}(\omega)$, the complex refractive index $n(\omega)=n_{r e}(\omega)+i n_{i m}(\omega)$ is given by ${ }^{6}$

$$
n(\omega)=\sqrt{|\epsilon(\omega)||\mu(\omega)|} \exp \left(\frac{i}{2}\left[\operatorname{arccot}\left(\frac{\epsilon_{r e}(\omega)}{\epsilon_{i m}(\omega)}\right)+\operatorname{arccot}\left(\frac{\mu_{r e}(\omega)}{\mu_{i m}(\omega)}\right)\right]\right)
$$

With the abbreviation $\eta_{j}=\mu_{j}$ for TE polarization and $\eta_{j}=\epsilon_{j}$ for TM waves, the continuity conditions $(6,7)$ for the interface between layers $j$ and $j+1$ can be written as

$$
F_{j}\left(z_{j}\right)=F_{j+1}\left(z_{j}\right), \text { and } \frac{1}{\eta_{j}} \frac{\partial F_{j}}{\partial z}\left(z_{j}\right)=\frac{1}{\eta_{l+1}} \frac{\partial F_{j+1}}{\partial z}\left(z_{j}\right)
$$

These conditions lead to a system of equations that relates amplitudes in neighboring layers through the step matrix

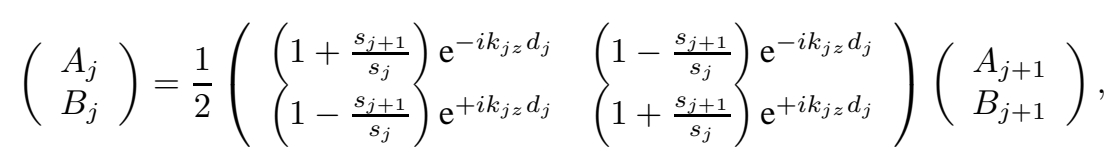


with the abbreviation $s_{j}=k_{j z} / \eta_{j}$ and where the separate propagation of the directional waves throughout the layers of thickness $d_{j}=z_{j}-z_{j-1}$ according to equation (8) has already been incorporated. Ordered multiplication of these matrices connects amplitudes in each layer of the structure. If the amplitude transfer is carried out over the full layer stack, one arrives at a system matrix of the form

$$
\left(\begin{array}{c}
A_{0} \\
r A_{0}
\end{array}\right)=\left(\begin{array}{ll}
m_{11} & m_{12} \\
m_{21} & m_{22}
\end{array}\right)\left(\begin{array}{c}
t A_{0} \\
0
\end{array}\right)
$$

Here $r$ and $t$ are the reflection and transmission amplitude coefficients. Further we define the transmittance as the ratio of the optical input and output power ${ }^{21}$ (intensity ratio for observation planes parallel to the layer surface)

$$
T(\omega, \theta)=\frac{n_{N+1} \cos \theta_{N+1}}{n_{\text {in }} \cos \theta_{0}}\left|\frac{1}{m_{11}}\right|^{2}
$$

and the reflectance as the ratio between the reflected and the incident power

$$
R(\omega, \theta)=\left|\frac{m_{21}}{m_{11}}\right|^{2} .
$$

Here it is assumed that the input and the output regions consist of the conventional dielectric materials without absorption. A certain portion of the optical power will be lost in the structure due to material absorption. For given reflectance $R$ and transmittance $T$, the absorptance of the structure is determined by the energy balance equation ${ }^{1} A=1-R-T$. In this paper, input and output medium are assumed to be without absorption and are modeled by real material parameters. Therefore, expressions for transmittance (16) and reflectance (17) are well defined.

\subsection{Negative index metamaterials}

Negative refractive index metamaterials are artificial composites, characterized by subwavelength features and negative real part of the refractive index of the homogenized structure. ${ }^{3-6}$ Usually they are made of the ordered arrangement of unit cells "atoms" that furnish effective electromagnetic response functions permittivity and permeability with designed properties. The key element is that these elementary electric and magnetic "particles" are subwavelength with respect to the target wavelength range. This leads to use of the homogenized effective response functions in the same fashion as in the natural materials and Maxwell's equations for media with the linear constitutive relations. Subwavelength features are not resolvable for the incident radiation and true effective medium approximation is physically founded. This is in contrast to other types of the artificial structures and media introduced recently in optics such as 2D and 3D Photonic Band-Gap materials, where the features are in the order of the wavelength, thus representing mesoscopic entities. The use of homogenized effective electromagnetic response functions in these structures has a rather loose physical foundation, although as an approximation for numerical and qualitative investigations ${ }^{19}$ can be useful.

All causal materials are bound to be dispersive, because the electric and magnetic polarizations depend on the history of the applied fields, thus responding non-instantaneously to their influence. ${ }^{3}$ Permeability and permittivity are in general complex functions of the frequency where the imaginary parts are related to dissipative processes in the material. Real and imaginary parts of the permeability and permittivity are connected via Kramers-Kronig relations. ${ }^{21}$

Dispersion in the NIM have to be present to ensure positive-definiteness of the energy density and necessarily is accompanied by losses due to Kramers-Kronig relations. ${ }^{3,6}$ In this paper we choose NIM part with Drude-type dispersion:

$$
\epsilon(\omega)=1-\frac{\omega_{p e}^{2}}{\omega\left(\omega+j \Gamma_{e}\right)}, \text { and } \mu(\omega)=1-\frac{\omega_{p m}^{2}}{\omega\left(\omega+j \Gamma_{m}\right)},
$$

where $\omega_{p e(m)}$ are the electric and magnetic plasma frequencies and $\Gamma_{e(m)}$ electric (magnetic) damping factors (usually expressed as a fraction of the plasma frequency). This choice is one of many available for modeling realistic NIMs. It represents a model of artificial electric and magnetic plasma. ${ }^{6}$ With use of (12) complex refractive index can be obtained.

Important feature of multilayers and other structures with NIM is the phase compensation or the process of the partial or the full removal of the waves phase shift after propagating through consecutive PIM and NIM layers. It is a consequence of the sign reversal in the phase factors $\delta_{j}=k_{j z} d_{j}$ in (14) for the propagating fields in the layers with NIM. ${ }^{8-10}$ For general multilayer containing NIM, the phase compensation influences greatly specific interference pattern and modify spectral transmission and reflection properties. In fact, all unusual properties associated with NIM-based structures arise from this effect. 


\subsection{Thermal radiation}

The electromagnetic energy emitted from the material bodies associated with its temperature and originating from heating processes inside material is called thermal radiation. It represents fundamental physical process of radiative energy transfer originating in microscopic processes of electromagnetic radiation emission induced by electron transitions in atoms, phonon transitions associated with molecular rotational and vibration modes and crystal lattice oscillations. In terms of wavelengths, it covers the ultraviolet spectrum, visible light spectrum and infrared spectrum.

Important concept necessary when dealing with the thermal radiation is the blackbody, i.e. an object that absorbs all incident radiation. The blackbody radiated power spectral distribution (for the EM radiation in thermal equilibrium at macroscopic temperature $T$ and frequency $\omega$. For the homogeneous, isotropic space is described by the Planck law

$$
\rho^{B B}(\omega, \beta)=\frac{\omega^{2}}{2 c^{2}} \frac{\hbar \omega}{\mathrm{e}^{\hbar \omega \beta}-1}
$$

where $\beta=1 /\left(k_{B} T\right)$ and $k_{B}$ is Boltzmann constant and $\hbar$ reduced Planck constant. Investigation of physical processes associated with thermal radiation in nature an engineering applications is important part of both theoretical and applied science.

Here, we are interested in classical waves phenomena associated with the electromagnetic waves, representing thermal radiation. We treat problem classically: with macroscopic Maxwell equations and given macroscopic material response functions permittivity, permeability and refractive index. All quantum-physical foundations and features are taken implicitly.

Phenomenological description for interaction between thermal radiation and matter includes process of reflection, transmission and absorption. The blackbody posses unit absorptivity (emissivity) and hence emits radiation with the same spectral distribution for the given temperature. This is a consequence of the Kirchhoff law: for the material object in thermal equilibrium with its surrounding spectral emissivity and absorptivity are equal for given temperature and every frequency, direction and polarization. This law can be obtained from thermodynamical principle of the detailed energy balance for equilibrium state that imposes equality between amount of energy being absorbed and emitted per frequency for given temperature. ${ }^{1}$ Thermal equilibrium of the whole system dictates energy conservation and with the Kirchhoff law imposes the relation between following macroscopic quantities:

$$
E(\omega)=A(\omega)=1-R(\omega)-T(\omega)
$$

Then, the emissivity can be obtained by multiplying blackbody power spectrum from Planck's law (19) by emittance:

$$
\rho(\omega, \beta)=E(\omega) \rho^{B B}(\omega, \beta)
$$

Equation (20) is fundamental for analysis of the emissive properties of the material objects and establishes the indirect method for the computing the thermal emission properties of arbitrary material objects via computing absorption of the object.

All real material objects posses emissive properties different from perfect blackbody due to non-ideal material properties. Various engineering applications dictate a necessity to alter these properties, for instance to approach the blackbody limit utilizing real materials.

Thermal radiation as considered here may have two different polarizations with the emittance $E_{T E}$ and $E_{T M}$ for TE and TM polarizations respectively. Unpolarized thermal radiation is assumed two have equal portions of both polarizations $E=1 / 2\left(E_{T E}+E_{T M}\right)$. We consider both polarized and unpolarized emittance.

\subsection{Multilayer structures as passive filtering systems for thermal radiation control}

We are interested in tailoring the spectrum of thermal radiation emitted by a thick substrate. This is achieved by depositing a thin-film multilayer structure on the top of the substrate. This structure simultaneously performs the functions of spectral filtering and angular redistribution of thermal radiation. To achieve this goal, we can use the approach based on an indirect and phenomenological method, as described previously. Figure 2 shows a general passive NIM-containing multilayer filter for thermal radiation control. It consists of a combination of positive and negative refractive index strata. This multilayer 


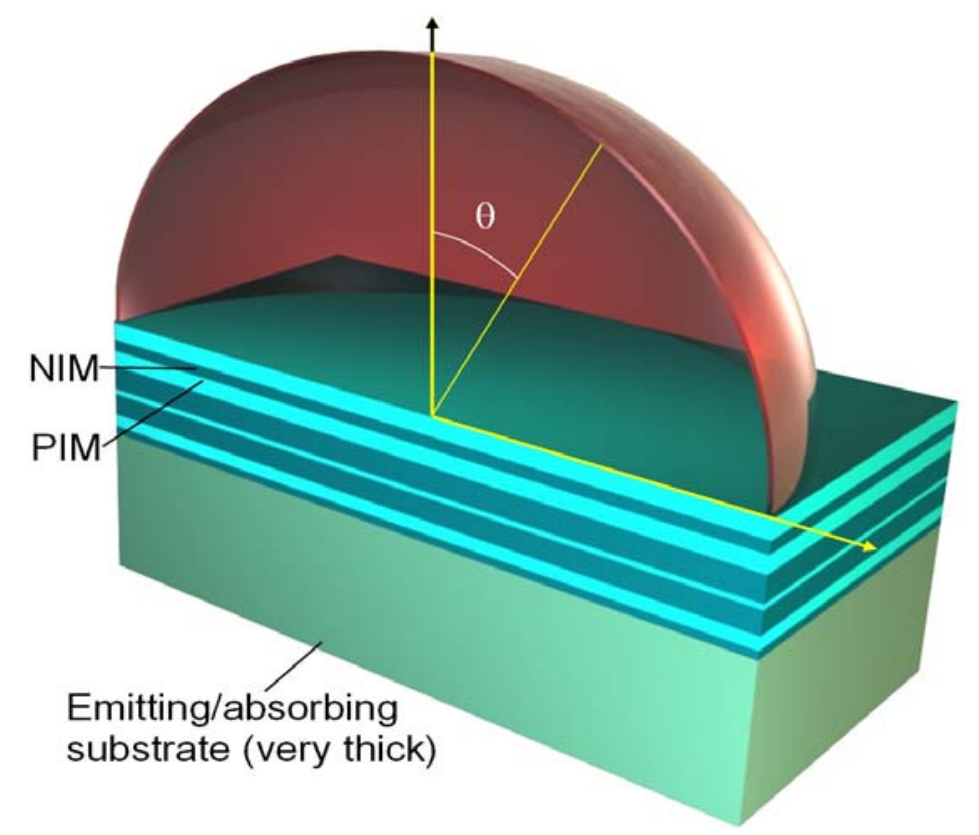

Figure 2. A general passive multilayer filtering system for thermal radiation control. The multilayer positioned on the top of thick absorbing substrate enable spectral and angular distribution shaping functionality. The ordering of materials in the multilayer is arbitrary and depends on concrete design example.

may be periodic, quasi-periodic or aperiodic and may contain one or more defect layers. It is deposited onto a relatively thick emitting /absorbing substrate.

The theoretical foundations for photonic bandgap (PBG)-based thermal radiation control have been outlined in. ${ }^{1}$ Periodic multilayer structures with an intrinsic photonic bandgap alter the thermal radiation spectrum by modifying the photonic density of modes. Thermal radiation is suppressed at frequencies inside the PBG, and enhanced at the frequencies of transmission resonances. In this way spectral redistribution of thermal power is achieved. This enables a control over thermal emission processes which is readily implemented by the available thin-film technologies.

Approach to the computation of the modified spectral emittance is simply and straightforwardly achieved through the Transfer Matrix Method. ${ }^{1}$ One can use it to obtain the dependence of absorption on the frequency and the angle of incident radiation. This method has been applied in literature to investigate the possibilities of thermal radiation control with the 1D, 2D and 3D PBG structures, ${ }^{16}$ as well as to achieve some particular design goals. The method enables thermal radiation control by tailoring the transmittance properties of multilayers which are applied as spectral and angular filters.

\section{RESULTS AND DISCUSSION}

Thermal radiation control with NIM-based multilayers has been considered in. ${ }^{7-9}$ It demonstrated a wider region of suppressed spectral emittance inside the photonic bandgap and a flatter spectral emittance in the band-edge region. Periodic PIM-NIM structures exhibit lower angular dependence of emittance than their all-positive counterparts. Another approach is to utilize periodic structures with one or more defects. The introduction of larger number of defects increases the complexity of the spectrum and increases the degree of freedom when tailoring the thermal radiation properties of the substrate. Besides periodic structures with or without defects, ${ }^{7}$ spectral and angular distribution of thermal radiation can be effectively modified by utilizing various quasiperiodic and aperiodic structures including pre-fractal Cantor multilayers ${ }^{8}$ quasi-periodic Fibonnaci-type multilayers, ${ }^{9}$ aperiodic Thue-Morse multilayers ${ }^{9}$ and dual-periodic multilayers. ${ }^{9}$ All of these introduce additional degrees of freedom through their special properties. For instance, the use of pre-fractal Cantor NIM-containing multilayers enables emittance superdirectivity and spectral selectivity.

The control over both spectral and angular spectrum in this case is achieved with generic design structures that shows robustness to structural disorder greater than that in conventional structures. In ordinary all-positive structures a simulta- 
neous control over spectral and angular distribution can be achieved by fabricating two separate tailoring structures, one for each of these goals.

Note that no surface plasmon-polariton modes interacting with the incident plane wave and influencing emittance spectra were taken into account. This is justified by the fact that plane wave can not excite SPP-s without additional mechanism (such as interface surface corrugation, assisting grating etc.) for photon momentum transfer, regardless of its incidence angle and frequency. ${ }^{3}$

To show basic principles and emphasize the effect of the NIM more clearly first we use frequency independent material parameters. Although, this is somewhat artifical with respect to current technology for NIMs, it is a usual choice for most of literature. To make our analysis more realistic we use also Drude type dispersion in the NIM part with losses included. This introduces very strong dispersion that is characteristic of the most metallic based systems. However, the best obtainable results concerning dispersion and losses in the NIM can be expected somewhere in between these two extremes.

\subsection{Single-defect PIM-NIM multilayer}

First example utilizes a periodic structure containing NIM and PIM with a single defect. We denote the PIM stratum as P and the NIM as N, while D is a defect which may be either PIM or NIM, but we chose in considered examples to be of the PIM type; $\mathrm{S}$ is the substrate. The structure is a quarter-wavelength multilayer coded as $N(P N){ }^{M_{1}} D(N P)^{M_{1}} N S$ with constant refractive indices $n_{N}=-1.5\left(\epsilon_{N}=2.25, \mu_{N}=-1\right), n_{P}=2\left(\epsilon_{P}=4, \mu_{P}=-1\right), n_{D}=n_{P}, n_{S}=3+i 0.3$ and $M_{1}=8$. Thicknesses of layers are chosen to be quarter-wavelength $n_{N} d_{N}=n_{P} d_{P}=\lambda_{0} / 4$ for target wavelength $\lambda_{0}$, while defect layer are half-wavelength $n_{D} d_{D}=\lambda_{0} / 2$. Frequency is expressed in terms of reduced frequency $\Omega=\omega / \omega_{0}$. Thickness of the absorbing layer (the last layer in the sequence) is $\operatorname{Re}\left(n_{S}\right) d_{S}=10 \lambda_{0}$. It is chosen here to be relatively thin when compared with the wavelength, although in the most of literature this thickness is much larger then the wavelength. Our choice emphasizes possibility for designing more compact device.
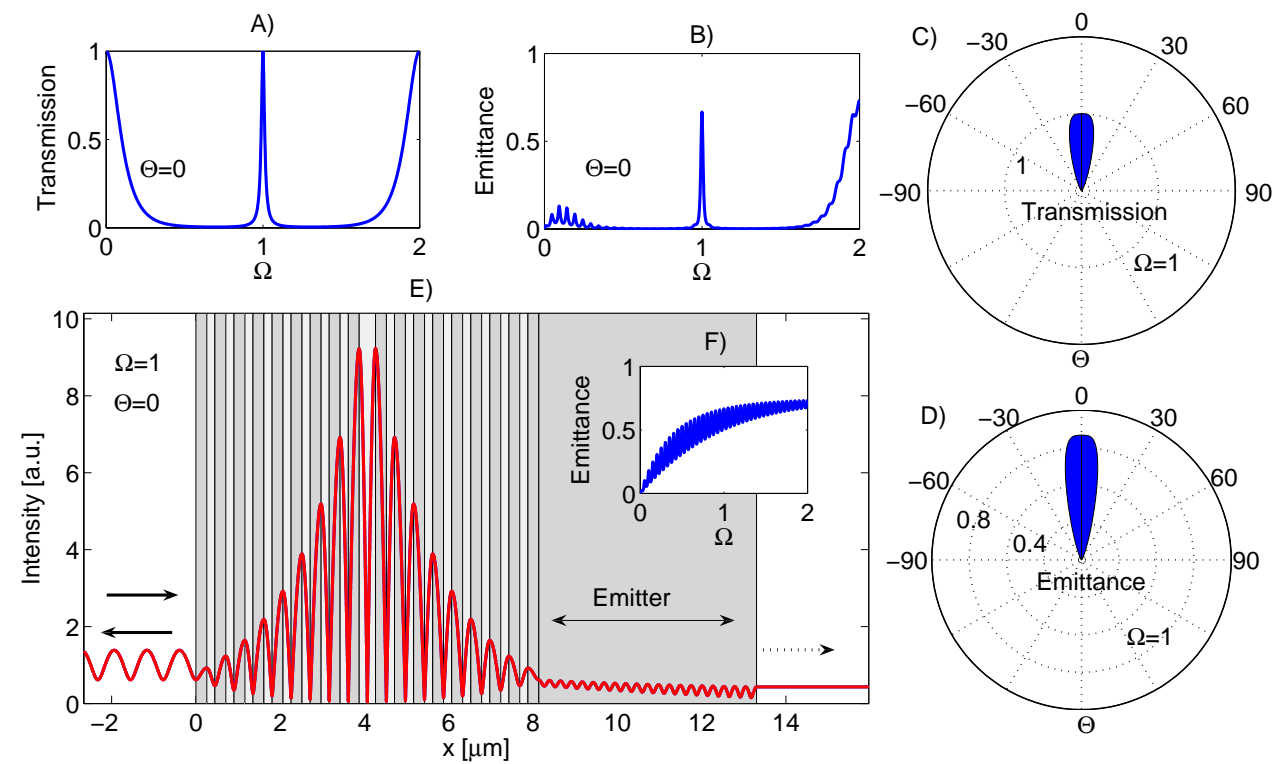

Figure 3. Selective and directional single defect PIM-NIM multilayer as resonant filter for the emittance shaping. Frequency dependent transmission A) and emittance B) for normal incidence $\Theta=0$. Angular dependence of the transmission C) emittance B) for the defect mode frequency $\Omega=1$. Field intensity for normal incidence and defect mode frequency $E$ ) and frequency dependence of substrate emittance without filter $F$ ).

Fig. 3A) shows the spectral transmission of the single defect-containing PIM-NIM structure for the normal incidence case. A single prominent and well defined defect mode is readily observable. The spectral emittance for normal incidence, shown in Fig. 3B), behaves in a similar fashion. Figs. 3C) and 3D) show the angular dependence of transmission and emittance at the defect frequency, where the strong angular directivity is clearly observable. This effect is the basis of the applicability of defect-containing PIM-NIM structures as thermal antennas. Fig. 3E) shows the spatial distribution of 

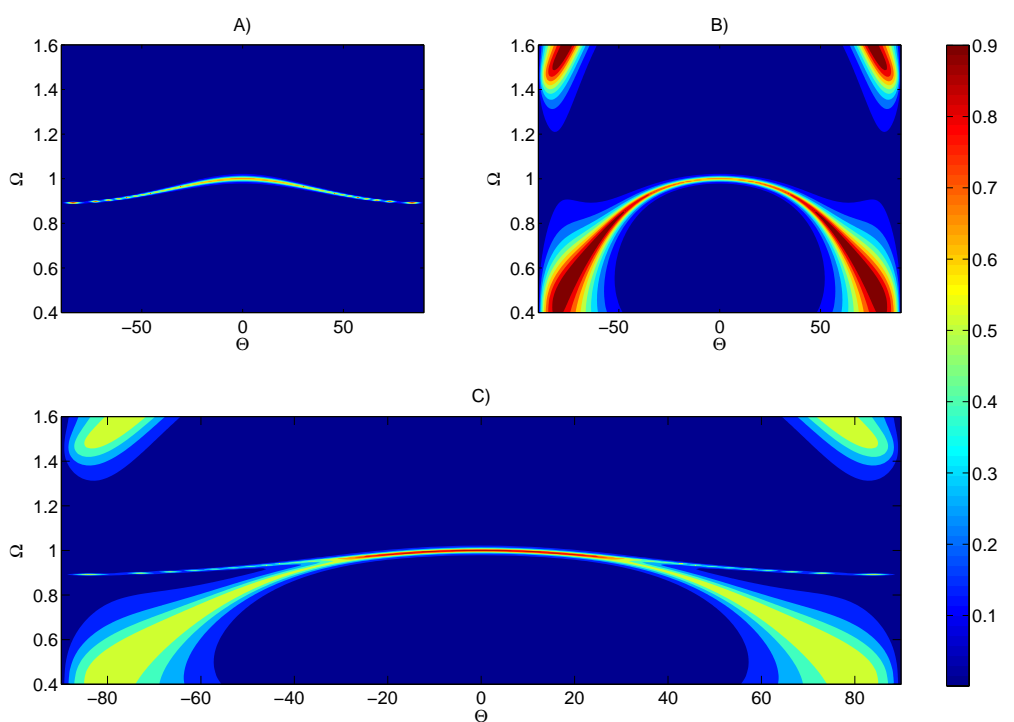

Figure 4. Frequency and angle dependence of the emittance for the emitter with single defect PIM-NIM multilayer as thermal radiation filter. Emittance for TE- mode radiation $A$ ) and TM-mode radiation B. Unpolarized emittance $C$ ).
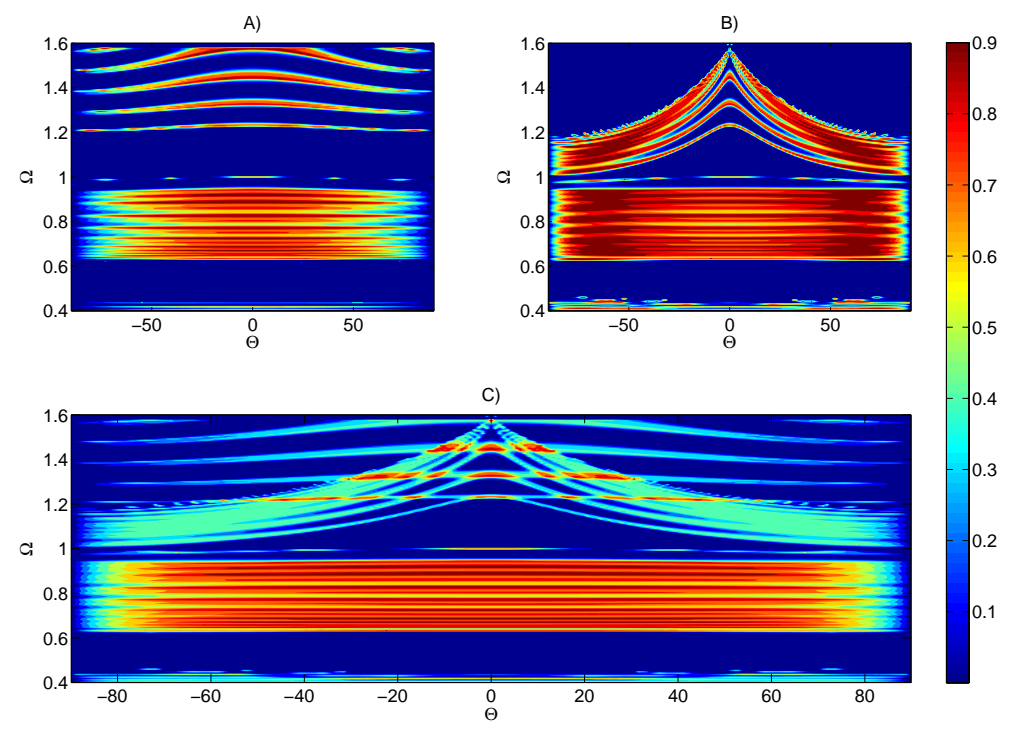

Figure 5. Frequency and angle dependence of the emittance for super-selective and highly-directional emitter with defect based PIMNIM multilayer; NIM part is taken to be dispersive and lossy with $n_{N}=1-2.5 \Omega^{2}+i 10^{-6}$; other parameters are the same as previous.

light intensity within the PIM-NIM multilayer for the frequency of the maximum of emittance, superposed on a drawing of the structural geometry of the multilayer-substrate system. It shows characteristic field localization in the vicinity of the defect. Note that the incident filed is not attenuated completely in the absorptive substrate that would happen if the thickness of the absorbing layer is chosen to be much bigger. The inset $(\mathrm{F})$ shows the spectral distribution of emittance if no multilayer NIM-PIM filter is applied. When compared with the Fig. 3A) it points out radical change of the emittance when the filter is applied. Frequency and angle dependence of the emittance for TE- mode ) and TM-mode radiation and unpolarized emittance is shown in Fig. 4. 
We choose parameters in (18) as $\omega_{p e}=\omega_{p m}=\omega_{p}$ and $\Gamma_{e}=\Gamma_{m}=\Gamma=10^{-6} \omega_{p}$. Refractive index of the NIM part then reads:

$$
n(\omega) \approx 1-\frac{\omega_{p}^{2}}{\omega^{2}}+i 10^{-6}
$$

where, we neglect frequency dependence in the imaginary part. This choice is somewhat arbitrary for our analysis and it has been used earlier by us ${ }^{7,8}$ and other authors. Consequence of the same frequency dependence for both permeability and permittivity in the NIM layers enforces condition of the perfect matching between the vacuum and NIM, and thus support reflection free propagation. However, in complex multilayer structures with combined dielectric materials and NIMs such as we analyze this condition is not satisfied between all layers and the multiple reflections in the multilayer occur.

Fig. 5 shows the influence of strong dispersion and losses in the case of a single-defect PIM-NIM structure. The dispersion and losses within the NIM part are described by a refractive index in the form $n_{N}=1-2.5 \Omega^{2}+i 10^{-6}$. Parameters for the NIM part are chosen in a such way that the physical thickness is given according to the condition $\left|n_{N}\right| d_{N}=\lambda_{0} / 4$ for $n_{B}(\Omega=1)=-1.5$, while the parameters in the PIM part are the same as before. The spectral and angular dependence of such a multilayer are shown in Fig. $5 \mathrm{~A}$ for TE polarization, in Fig. $5 \mathrm{~B}$ for TM and in Fig. $5 \mathrm{C}$ for the unpolarized case. The distinct defect modes are still observable inside the bandgap region. However, dispersion in the refractive index introduces more complex scattering pattern in such way that additional frequencies where emittance is enhanced are present. The introduction of the dispersion and losses in the NIM part shrinks the underlying bandgap region same as in the previous example with the single defect structure. However, the designed functionality is preserved.

\subsection{Multiple defect PIM-NIM multilayer}

In this example we use a filter design for multiple-channeled narrow-band emittance. To this purpose additional defects are introduced into a periodic PIM-NIM multilayer structure. Similar to the all-positive case, this produces a spectrally multiple-channeled emittance with a narrow angular dependence, while retaining benefits of the widened bandgap region of the periodic PIM-NIM multilayer.

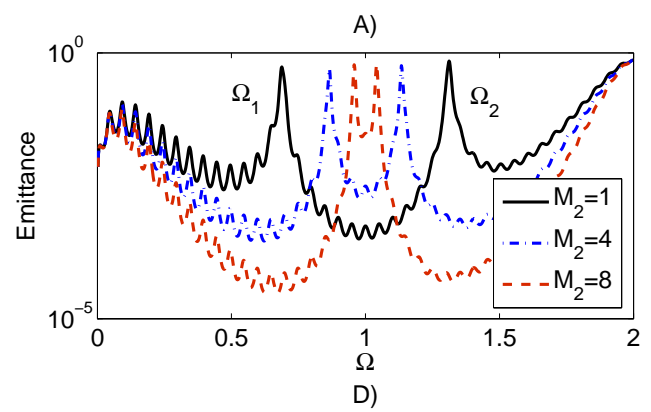

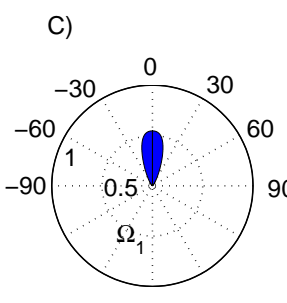

$\Theta$
D)

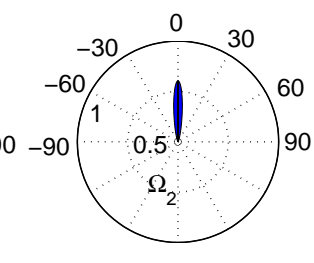

$\Theta$

E)

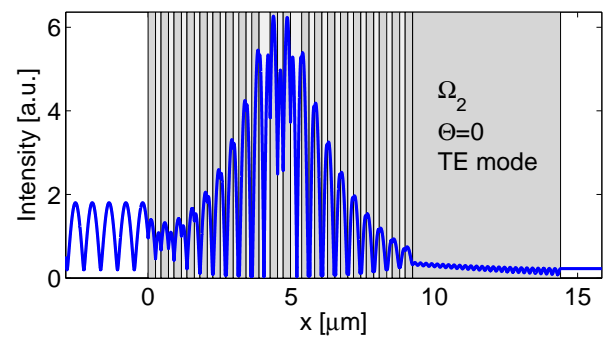

Figure 6. A) Emittance for multiple defect PIM-NIM multilayer with different separation of the defects. Angle dependence of the emittance B)-C) for TE polarization for resonant frequencies $\Omega_{1}$ and $\Omega_{2}$. TE-mode field intensity distribution D) and E) for resonant frequencies $\Omega_{1}$ and $\Omega_{2}$ respectively.

The antenna-like behavior for the multiple defect case is observed in Fig. $6 \mathrm{~A}$ ) showing the calculated spectral emittance under normal incidence for a structure with two defects coded as $N(P N)^{M_{1}} D(N P)^{M 2} N D(N P)^{M_{1}} S$ where $M_{1}=8$ and $M_{2}$ is varied. Two prominent peaks are visible, corresponding to the two defect transmission modes supported by the structure. Thus, antenna-like behavior of the emitting substrate can be further exploited in the NIM case by introducing 

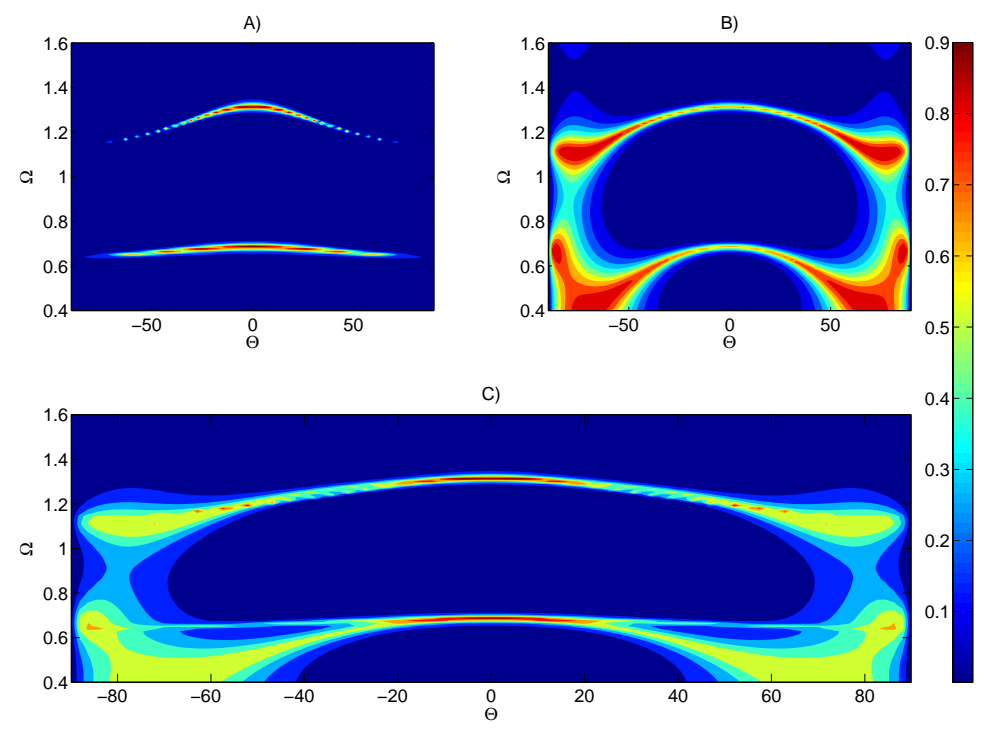

Figure 7. Frequency and angle dependence of the emittance for the emitter with multiple defect PIM-NIM multilayer as thermal radiation filter. Emittance for TE- mode radiation A) and TM-mode radiation B. Unpolarized emittance $C$ ).

multiple defects. Directional diagrams of the antenna, i.e. polar graphs of the emittance for TE polarization and for two characteristic frequencies are shown in Fig. 6B)-C). High directionality is present with two well separated frequency channels. Angular emittance is calculated for two different resonance frequencies, $\Omega_{1}=0.6815$ and $\Omega_{2}=1.315$ and TE polarization. The angular and spectral filtering, resulting in high directivity, are present and it can be tuned through the choice of multilayer parameters. Here, their existence is influenced by the existence of the two defect modes inside the wide bandgap region. Field intensity distributions for two characteristic frequencies corresponding to the maxima of the emittance are shown in Fig D) and E).

Frequency and angle dependence for the emittance with TE and TM polarizations and unpolarized case are shown in Figure 7. Two distinct defect modes are readily observed inside the bandgap region. However, introduction of the dispersion and losses in the NIM part (not shown here) shrinks the underlying bandgap region same as in the previous example with the single defect structure.

\section{CONCLUSIONS}

The use of NIM for thermal radiation control offers some advantages over positive-index PBG structures. 1D NIMcontaining structures render a unique possibility to obtain spectrally selective thermal emitters with both narrow and wide angular fields of view. The latter requires a careful design with respect to spectral and angular dependence shaping, regardless of the structure dimensionality, dispersion and absorptive characteristics. Simple design criteria for NIM-containing multilayers are sufficient to obtain the same type of functionality owing to phase compensation effect associated with the NIM.

Following previous results for thermal radiation control by periodic ${ }^{7}$ and non-periodic multilayers containing NIM, ${ }^{8,9}$ we show designs for both spectral selectivity and directionality of the emittance utilizing NIM-containing multilayers designed as finite periodic sequences with structural defects.

As for multilayers based on the ordinary materials suitably built-in defect in otherwise periodic structure gives rise to a resonance inside the bandgap. At the same time wide bandgap region (specific feature of the NIM-containing multilayers), covering almost all frequencies, is retained. Inclusion of the multiple defects leads to a multiple-channeled thermal emittance. Scalability and robustness to small-scale disorder are some additional benefits that can be expected by the novel design of thermal antennas. Moreover, we demonstrated that introduction of the large dispersion and absorption in the NIM part itself does not destroy the designed features. 
Some special features not achievable by the conventional all-positive index structures may arise when the filtering structure containing both NIM and PIM strata is designed to operate within the zero-n bandgap regime. Although, zero$\mathrm{n}$ bandgap regime requires additional analysis, in this mode of operation it might be possible to render rejection bands which are wide both in the spectral and the angular domain, or, alternatively, ultra-narrow pass-bands with angular superselectivity.

With the prospect of the NIMs operating for optical frequencies and with controllable intrinsic dispersion and absorption properties thermal radiation antennas utilizing NIM-containing structures can be seen as promising design choice.

\section{Acknowledgment}

This work is financially supported by NanoNed, flagship NanoPhotonics, project TOE.7143. Z. Jaksic acknowledges support by the Serbian Ministry of Science within the framework of the project IT.6151.B.

\section{REFERENCES}

1. C. M. Cornelius and J. P. Dowling, "Modification of planck blackbody radiation by photonic band-gap structures," Phys. Rev. A 59(6), pp. 4736-4746, 1999.

2. S. A. Ramakrishna, "Physics of negative refractive index materials," Rep. Prog. Phys. 68, pp. 449-521, Jun 2005.

3. P. W. Milonni, Fast Light, Slow Light and Left-Handed Light, IOP Publishing, 2005.

4. N. Engheta and R. W. Ziolkowski, Metamaterials: Physics and Engineering Explorations,, Wiley-IEEE Press, 2006.

5. Z. Jaksic, N. Dalarsson, and M. Maksimovic, "Negative refractive index metamaterials: Principles and applications," Microwave Review 12(1), pp. 36-49, 2006.

6. K. Busch, G. von Freymann, S. Linden, S. F. Mingaleev, L. Tkeshelashvili, and M. Wegener, "Periodic nanostructures for photonics," Phys. Rep. 444, 2007.

7. M. Maksimovic and Z. Jaksic, "Modification of thermal radiation by periodical structures containing negative refractive index metamaterials," Phys. Lett. A 342, pp. 497-503, 2005.

8. M. Maksimovic and Z. Jaksic, "Emittance and absorptance tailoring by negative refractive index metamaterial-based cantor multilayers," J. Optics A: Pure and Applied Optics 8(3), pp. 352-362, 2006.

9. F. F. de Medeiros, E. L. Albuquerque, M. S. Vasconcelos, and P. W. Mauriz, "Thermal radiation in quasiperiodic photonic crystals with negative refractive index," J. Phys.: Condens. Matter 19(496212), 2007.

10. L. Feng, X.-P. Liu, M.-H. Lu, and Y.-F. Chen, "Phase compensating effect in left-handed materials," Phys. Lett. A 332(5-6), pp. 449-455, 2004.

11. J. Li, L. Zhou, C. T. Chan, and P. Sheng, "Photonic band gap from a stack of positive and negative index materials," Phys. Rev. Lett. 90(083901-1), 2003.

12. F. F. de Medeiros, E. L. Albuquerque, and M. S. Vasconcelos, "Optical transmission spectra in quasiperiodic multilayered photonic structure," J. Phys.: Condens. Matter 18, pp. 8737-8747, 2006.

13. J. Monsoriu, R. A. Depine, and E. Silvestre, "Non-bragg band gaps in 1d metamaterial aperiodic multilayers," J. Eur. Opt. Soc. 2(07002), 2007.

14. A. Narayanaswamy and G. Chen, "Thermal emission control with one-dimensional metallodielectric photonic crystals," Phys. Rev. B 70(125101), 2004.

15. F. OSullivan, I. Celanovic, N. Jovanovic, J. Kassakian, S. Akiyama, and K. Wada, "Optical characteristics of onedimensional Si/SiO2 photonic crystals for thermophotovoltaic applications," J. Appl. Phys. 97(033529), 2005.

16. M. Florescu, H. Lee, A. J. Stimpson, and J. P. Dowling, "Thermal emission and absorption of radiation in finite inverted-opal photonic crystals," Phys. Rev. A 72(033821), 2005.

17. M. Florescu, K. Busch, and J. Dowling, "Thermal radiation in photonic crystals," Phys. Rev. B 75, p. 201101(R), 2007.

18. W. C. Chew, Waves and Fields in Inhomogeneous Media, IEEE Press, 1990.

19. J.-M. Lourtioz, Photonic Crystals: Towards Nanoscale Photonic Devices, Springer, 2005.

20. R. Ziolkowski and E. Heyman, "Wave propagation in media having negative permittivity and permeability," Phys. Rev. E 64(056625), 2001.

21. M. Born and E. Wolf, Principles of optics, Cambridge University Press, 2003. 